\title{
La recuperación mutua en personas con trastornos psicológicos o diversidad funcional a través de la práctica creativa
}

\section{Mutual recovery in people with psychological disorders or functional diversity through the creative practice}

\author{
F. JAVIER SAAVEDRA-MACÍAS \\ Universidad de Sevilla \\ fjsaavedra@us.es \\ SAMUEL ARIAS-SÁNCHEZ \\ Universidad de Sevilla \\ samuel@us.es \\ EYLIN DE LA CRUZ \\ Universidad de Sevilla \\ M. LuISA GALÁN \\ Universidad de Sevilla \\ BLANCA GaLVÁN \\ Universidad de Sevilla \\ Lara Murvartian \\ Universidad de Sevilla \\ Natividad Vallecillo \\ Universidad de Sevilla
}

\section{Resumen}

En el presente artículo introducimos el concepto de "recuperación mutua" y proponemos las prácticas creativas como herramientas eficientesde recuperación de personas tanto con problemas de salud mental como con algún tipo de diversidad funcional. Frente al concepto clásico de "arte-terapia" nosotros proponemos el concepto de "práctica creativa" como más compatible con el modelo de "recuperación mutua". Para ello, en primer lugar realizamos un breve repaso crítico a la relación del arte con la locura. Seguidamente, presentamos los conceptos hermanos de "recuperación" y "recuperación mutua" en el marco de lo que se ha venido a denominar las "health humanities". Para finalizar, describimos dos prácticas creativas que en la actualidad están siendo evaluadas en España en el contexto de un proyecto de investigacióninternacional en recuperación mutua: Los seminarios creativos con personas con 
trastorno mental grave en el Museo de Arte Contemporáneo de Sevilla y el grupo de teatro con personas con diversidad funcional de la Asociación Síndrome de Down-Sevilla.

Palabras clave: prácticas creativas, arte-terapia, salud mental, trastorno mental grave, recuperación mutua.

Saavedra-Macías, F.J., Arias-Sánchez, S., de la Cruz, E., Galán, M.L., Galván, B., Murvartian, L., Vallecillo, N. (2016): La recuperación mutua en personas con trastornos psicológicos o diversidad funcional a través de la práctica creativa . Arte, Individuo y Sociedad, 28(2) 339-354

\title{
Abstract
}

In this paper we introduce the concept of "mutual recovery" and we propose creative practices as efficient recovery tools people with both mental health problems as some sort of functional diversity. Compared with the traditional concept of "art therapy" we propose the concept of "creative practice" as more consistent with the model of "mutual recovery." To do this, we first make a brief critical review of the relationship between art and madness. Next, we present the related concepts of "recovery" and "mutual recovery" in the context of what has become known as the "health humanities". Finally, we describe two creative practices currently being tested in Spain in the context of the a international research project about mutual recovery:Creative workshops with people with serious mental illness at the Museum of Contemporary Art of Seville and the theater group of people with diverse functionality with the Down Syndrome Association of Seville. Keywords: creative practices, art-therapy, mental health, serious mental illness, mutual recovery.

Sumario: 1. Introducción: Una breve mirada a la historia del Arte y la Salud Mental, 1.1 ¿Es la locura propia de los artistas?, 1.2 ¿Puede convertirse el Arte en una herramienta terapéutica?, 2. El modelo de la "Recuperación": Una nueva aproximación a la Salud Mental, 3. La práctica creativa como "Recuperación Mutua", 4. Dos ejemplos de Práctica creativa como recuperación mutua, 4.1. Talleres creativos en el Museo de Arte Contemporáneo de Sevilla, 4.2. Actividad del grupo de teatro ASEDOWN, 5. Reflexión final. Referencias.

Este artículo recoge resultados de la investigación Creative Practice as Mutual Recovery: Connecting Communities for Mental Health and Well-Being liderado por "Centre Social Futures" del "Mental Health Institute" de Nottingham y financiada por el Royal Arts and Humanities Council.

\section{Introducción: Una breve mirada a la historia del Arte y la Salud Mental}

\author{
“Claro que este arte está loco ¿Qué arte no es loco? Cuando no está loco no es
} arte" (Dubuffet).

Si echamos la mirada atrás podemos observar una relevante y antigua preocupación por la salud mental y las prácticas creativas. Este interés ha sido y es propio tanto de especialistas en disciplinas relacionadas con las ciencias de la salud (psicólogos o psiquiatras) como por artistas o especialistas en historia del arte. Nosotros vamos a intentar describir distintas formas de afrontar la relación entre la práctica artística y la salud mental.Estas tipologías las podríamos categorizar de acuerdo con las preguntas que los expertos y autores han pretendido contestar o explorar: ¿Es la locura propia de los artistas? Y ¿Puede convertirse el Arte en una herramienta terapéutica? 


\section{1. ¿Es la locura propia de los artistas?}

Aunque el interés por la relación entre locura y arte es muy evidente en el siglo XIX y desemboque en el siglo XX con las vanguardias artísticas, éste viene de lejos. Sin querer hacer un repaso exhaustivo, por ejemplo Platón asocia la locura con la inspiración divina en su obra Fedro. Aunque eso sí, conocida su animadversión por los poetas, sitúa esa locura correspondiente al arte muy por debajo de otros tipos de locura. De hecho, sitúa a los poetas por debajo de los maestros de gimnasio y los adivinadores. En el Renacimiento se consolida la figura del autor, del artista, junto con una concepción del "yo" totalmente moderna. Es decir, el "Yo" se entiende como autónomo, agente, y poseedor de un mundo independiente y diferenciado. No es por tanto extraño que nazca la figura del creador aislado, atormentado por sus inquietudes y sus pasiones, las cuáles no puede controlar y a la vez lo hacen genial. Quizás una de las imágenes más logradas de esta figura corresponde al grabado de Durero "Melancolía". De hecho, esta figura de artista "melancólico" ha sobrevolado la cultura de Europa hasta nuestros días. El Romanticismoalemán llevará la hipertrofia del "Yo" hasta cimas sorprendentes. Fichte (1984),unos de los idealistas alemanes más influyentes, consideraba al "Yo" como creador del mundo incluyendo las leyes de la Naturaleza en la "Introducción a la teoría de la ciencia". No es extraño que esta premisa haya sido tildada de solipsismo, el cuáles compatible con el aislamiento y lucidez del artista demente y "melancólico".Imagen que, más allá del ámbito pictórico, transciende disciplinas artísticas y que a su vez representa a compositores como el último Beethoven, Chopin o Schubert o poetas como Novalis y Hölderlin, y fundamenta los movimientos vanguardistas que abordan la renovación del arte.

Sin lugar a dudas, las vanguardias artísticas han hecho más accesible las obras de arte de autores que lejos de los círculos artísticos académicos o sociales han desarrollado alguna obra. Por ejemplo, el expresionismo en su viaje hacía la autenticidad en la expresión de las emociones toma como modelo el arte primitivo o indígena,libre de los valores decadentes de las sociedades burguesas. La búsqueda de lo espontáneo e inmediato, sin mediación, acerca también a estos autores a las producciones de las personas con problemas de salud mental. La influencia del surrealismo también es determinante en la búsqueda de técnicas que hagan sobresalir los procesos inconscientes y alejen el arte de la conciencia, demasiado compleja y mediada por la represión moral. Por ejemplo, mediante técnicascomo el automatismo pictórico. Esta necesidad de técnicas artísticas nuevas aproximó a los artistas a las experiencias de las personas con trastornos mentales graves (TMG).El dadaísmo,muy relacionado con el surrealismo se propuso huir de la lógica, de la razón y de los cánones establecidos y desembocó en prácticas artísticas hasta el momento inauditas, propias de dementes y de personas delirantes. Algunas corrientes artísticas de mediado de siglo XX han continuado defendiendo premisas similares.Por ejemplo el Arte marginal, después acuñado por Dubuffet como Art Brut, privilegia explícitamente como arte de especial interés el producido por personas con trastornos mentales o discapacidad. El interés por estos autores es debido a su carácter marginal que los desconecta de los condicionantes externos y asegura una motivación intrínseca.

Podríamos continuar con esta descripción. Somos conscientes de que hay periodos históricos que no hemos reseñado, la Edad Media, el Barroco, etc., sin embargo, 
nuestro objetivo es simplemente señalar algunos hitos que hemos considerado importantes en la relación entre el arte y la locura.

Finalmente nos interesa enfatizar algunos puntos en común entre gran parte de las vanguardias. La búsqueda, más o menos radical, de la fractura de cualquier condicionamiento social, estético o moral, la necesidad de una motivación intrínseca que asegure la autenticidad de la expresión y la huida de cualquier asociación lógica. Así, de una manera u otra, se termina por identificar todas estas características con la creación artística más original. ¿Y dónde encontrar mejor personas que cumplan estos requisitos que en un psiquiátrico? Desde esta posición no resulta extraño que se identifique la figura del artista verdadero al loco, al demente, al primitivo o incluso, al niño.

\section{2. ¿Puede convertirse el Arte en una herramienta terapéutica?}

Desde otra perspectiva, alienistas, y más tardes psiquiatras, muy pronto comenzaron a preguntarse por las creaciones artísticas de los pacientes a los cuáles trataban mediante un método científico o pseudocientífico. En el año 1864 Lombroso publica"Genio y Locura". En este ensayo Lombroso concluye que las producciones artísticas de los dementes no son más que una manifestación de su locura y que no cumplen ninguna función social. De otra forma lo sostuvo Fursac cuando afirmó que las producciones artísticas de los enfermos mentales no eran más que una manifestación concreta de su enfermedad (Gilman, 2001). Lombroso asemeja la necesidad de crear de las personas con locura con las compulsiones de los salvajes y primitivos. Como hemos visto, esta característicadel arte de las personas con locura sería considerada de gran valor por las vanguardias artísticas medio siglo más tarde. Merece la pena recordar que Lombroso fue uno de los precursores de la frenología criminológica al correlacionar las asimetrías craneales con tipologías de delincuentes. Y es que las creaciones artísticas de los residentes de los recientes hospitales psiquiátricos se convirtieron en instrumentos para categorizar y diagnosticar a personas.

El psiquiatra francés Max Simon en el último cuarto del siglo XIX halló características específicas en la estructura y la forma de las obras de los pacientes dependiendo de su diagnóstico: demencia, imbecilidad, melancolía, etc. Ya entrado el siglo XX, Jaspers (1913), desde una perspectiva fenomenológica alejada del biologicismo de Lombroso, consideraba que las obras de los internados en los hospitales psiquiátricos eran un instrumento para acceder a su yo esencial.

Prinzhorm, psiquiatra e historiador de arte alemán es una de las figuras más influyentes en este campo. En su paso por el Hospital de Heidelberg y con la ayuda de su director Karl Wilmmans llegó a reunir miles de obras de arte de personas con TMG. Todavía hoy en el citado hospital existe un museo abierto al público.

Prinzhorm publicó en 1922 "Bildnerei der Geisteskranken" (El arte de los enfermos Mentales) donde mediante el análisis de la obra de la colección y algunos casos de pacientes especialmente relevantes analiza las pulsiones que conducen a la práctica creativa de los pacientes. Aunque esta obra no era ajena a la cuestión de la relación entre los rasgos y las características plásticas de las obras de los pacientes y su sintomatología psicopatológica, al igual que sus antecesores, su foco lo situó 
en otro lugar. En "Bildnerei der Geisteskranken" se analizan las pulsiones básicas que constituíanla actividad artística de los pacientes. Pulsiones que pudieran explicar también la actividad artística de las personas sanas. En definitiva, Prinzhorm intenta descubrir los impulsos innatos de la creación artística mediante el análisis de las obras de los pacientes, ya que éstos, según el mismo, se encontraban libres de toda influencia cultural y académica. De nuevo, la figura del salvaje o primitivo. Es evidente que esta premisa es falsa, algunos pacientes que se reseñan en la obra incluso habían recibido formación artística. En este importante ensayo se señalan seis pulsiones: la expresión, el juego, el dibujo ornamental, la ordenación compulsiva de los elementos del dibujo, la copia obsesiva y la construcción de estructura simbólicas.

A pesar de que Prinzhorm evitaba identificar a sus pacientes con artistas, su teoría fue muy importante para las vanguardias artísticas, especialmente el expresionismo, y no fue extraño encontrar a partir de entonces comparaciones directas entre las obras de los pacientes y las obras de artistas. Por ejemplo, Schilder en 1918 comparó directamente un paciente que estudiaba con autores como Kandinsky (Gilman, 2001).

Este floreciente campo de investigación fue truncado trágicamente por el nazismo. Como ejemplo de esta ruptura, muchas de las obras de la Clínica Universitaria de Heidelberg fueron expuestas junto con las obras de artistas consagrados de las vanguardias en la exposición de Munich organizada por el gobierno Nazi llamada "Entertete Kunst" (Arte degenerado) en el año 1937.

Las prácticas artistas de pacientes se consideraban por los profesionales de la psiquiatría más un instrumento de diagnóstico o análisis de los conflictos internos que un medio terapéutico. Incluso Prinzhorm, el cual matiza esta preocupación por el diagnóstico al centrarse exclusivamente en las pulsiones artísticas, niega la capacidad de creación autónoma al anclarla en una necesidad interior de carácter irresistible incondicionada social o culturalmente.

En los años 40 del pasado siglo se acuña el término de arte-terapia por primera vez. Margaret Naumburg, Edith Kramer y Adrian Hill, entre otros, comienzan a utilizar el arte claramente como instrumento terapéutico. Naumburg utilizó el arte como instrumento clínico en su trabajo como terapeuta dentro del ámbito del psicoanálisis. Esta autora publicó en el año 1947 su libro "Art Expression of Behaviour Disturbed Children as a Means of Diagnosis and Therapy".Adrian Hill experimentó en primera persona los beneficios psicológicos del arte durante el tiempo que estuvo internado en un hospitaly en el año 1943 publica "Art as an aid to illness: an experiment in occupational therapy". Edith Kramer, que provenía de centro Europa y había observado los estragos que habían provocado la guerra y el holocausto en los niños, comienza a trabajar con niños en EEUU en el ámbito educativo. Estos autores, muchos de ellos influenciados por la corriente psicoanalista, fueron los precursores de la disciplina de la arte-terapia la cual un par de décadas después desembocó en la creación de las primeras sociedades de arte-terapia.

Veinte años más tarde y en un ámbito estrictamente sanitario Ronald David Laing fundó su famosa comunidad para personas con esquizofrenia de Kingsley Hallen, Londres, en el año 1965. Entre otras prácticas alternativas radicales se consideraba la expresión libre de las emociones por medios artísticos y la regresión a la infancia como procesos de sanación. Laing llegó a reconocer a los pacientes como mediadores 
sagrados que acercan el mundo real de las emociones al resto de los seres humanos, los cuales no disponemos de la visión interior de éstos. Mary Barnes, paciente que decidió alojarse en la comunidad de Laing tras leer su obra "El yo dividido" (1960), se convirtió en una reputada artista tras su paso por Kingsley Hallen.

En nuestra opinión, y como se puede deducir del pequeño camino que hemos recorrido, la relación entre las prácticas creativas y la salud mental ha estado encuadrada entre dos ejes. Por una parte, la imagen del artista genial enloquecido, y por otra, el contexto claramente clínico del uso instrumental del arte. El énfasis en el arte como instrumento de diagnóstico y la influencia del psicoanálisis es una muestra de ello.

En este sentido,pensamos que el concepto de arte-terapia y su práctica presentan una serie de rasgos que creemos constructivo superar. Primero, su connotación clínica de la actividad al ser conceptualizada como una terapia, quedando el impacto psicosocial escondido tras los aspectos individuales y reproduciendo el marco de experto-paciente. Segundo, el uso del término "arte" conlleva una serie de imágenes relacionadas con una visión sesgada, pero que persiste en la población, relacionada con la belleza, la genialidad y la autoría. Por último y fuera de este debate conceptual, en nuestra opiniónfalta evidencia empírica de su eficacia. Es decir, existe una carencia de utilización de diseños rigurosos, que no tienen por qué restringirse a métodos cuantitativos, para evaluar la eficacia de las intervenciones y que diferentes autores han reseñado (Reynolds, Nabors y Quinlan, 2011).

\section{2. ¿EI modelo de la "Recuperación": Una nueva aproximación a la Salud Mental}

El modelo de la recuperación ha supuesto una revolución en la forma de entender la enfermedad mental y cómo enfrentarse a ésta. Este impacto es determinante en los países anglosajones, donde su influencia se observa incluso en los diseños de los servicios sociales y sanitarios y en las prácticas profesionales. Aunque con mucha menos intensidad, este modeloya empieza a ser evidente en España y otros países del sur de Europa. Para entender el nuevo papel que las prácticas creativas pueden desempeñar en este nuevo modelo creemos necesario describirlo y definirlo brevemente. El Sainsbury Centre for Mental Health describe la recuperación de la siguiente manera:

Una serie de valores sobre los derechos de la persona a construir una vida significativa para sí misma, con o sin la presencia continua de sintomas de salud mental. La recuperación está basada en las ideas de la autodeterminación y el control de sí mismo. Hace énfasis en la importancia de laesperanza en el mantenimiento de la motivación y la ayuda con el objetivo de la consecución deuna vida llena de sentido (The Future Vision Coalition, 2009, p.22).

Al contrario de otros modelos esenciales en la historia de los servicios de salud mental, como el modelo de la "rehabilitación" o de "integración", el modelo de la recuperación tiene su origen en las experiencias de superación de los mismos afectados. En la tabla 1 se pueden observar las diferencias entre estos modelos. De hecho, los primeros impulsores de este modelo son los grupos de autoayuda y las asociaciones de "Víctimas del modelo psiquiátrico" como algunos se denominan. Es 
necesario remarcar que los rasgos del concepto de recuperación que se indican en esta tabla no son incompatibles con los distintos rasgos de los conceptos de rehabilitación e integración.

\begin{tabular}{cccc} 
& Rehabilitación & Integración & Recuperación \\
\hline $\begin{array}{c}\text { Disciplinas } \\
\text { relacionadas }\end{array}$ & $\begin{array}{c}\text { Aprendizaje social, } \\
\text { psicología, psiquiatría } \\
\text { social. }\end{array}$ & $\begin{array}{c}\text { Derechos } \\
\text { ciudadanos, procesos } \\
\text { estigmatizadores, } \\
\text { antropología, sociología. }\end{array}$ & $\begin{array}{c}\text { Psicología narrativa } \\
\text { psicología cultural, } \\
\text { antropología social y } \\
\text { cultural. }\end{array}$ \\
\hline
\end{tabular}

\begin{tabular}{|c|c|c|c|}
\hline Enfoque & Social e individual & Social y político & $\begin{array}{l}\text { Subjetivo, experiencias } \\
\text { personales, sociocultural. }\end{array}$ \\
\hline
\end{tabular}

\begin{tabular}{cccc}
\hline Objetivo & $\begin{array}{c}\text { Aprendizaje de } \\
\text { conductas reforzantes } \\
\text { socialmente. }\end{array}$ & $\begin{array}{c}\text { Ciudadanía, integración } \\
\text { social. }\end{array}$ & $\begin{array}{c}\text { Construcción identidad } \\
\text { coherente, logro del } \\
\text { significado personal. }\end{array}$ \\
\hline $\begin{array}{c}\text { Tipo de } \\
\text { intervención }\end{array}$ & $\begin{array}{c}\text { Programas de } \\
\text { carácterconductual. }\end{array}$ & $\begin{array}{c}\text { Gestión sociosanitaria, } \\
\text { programas de } \\
\text { sensibilización e } \\
\text { información, programas } \\
\text { laborales y residenciales. }\end{array}$ & $\begin{array}{c}\text { Influencia del entorno, } \\
\text { relaciones informales } \\
\text { o asistemáticas, } \\
\text { recursos para construir } \\
\text { significados, denuncia. }\end{array}$ \\
\hline Perspectiva & Molecular & Molar & Molar \\
\hline $\begin{array}{c}\text { Evaluación e } \\
\text { investigación }\end{array}$ & $\begin{array}{c}\text { Esencialmente } \\
\text { cuantitativa: } \\
\text { psicométrica y } \\
\text { observacional }\end{array}$ & Mixta & $\begin{array}{c}\text { Esencialmente cualitativa, } \\
\text { experiencia subjetiva, } \\
\text { narrativa. }\end{array}$ \\
\hline
\end{tabular}

\begin{tabular}{|c|c|c|c|}
\hline Protagonistas & $\begin{array}{c}\text { Profesionales del } \\
\text { área de la psicología o } \\
\text { psiquiatría }\end{array}$ & $\begin{array}{c}\text { Asociaciones de } \\
\text { familiares,grupos } \\
\text { políticos. }\end{array}$ & $\begin{array}{l}\text { Familiares, pacientes, } \\
\text { afectados en general, } \\
\text { clientes, supervivientes. }\end{array}$ \\
\hline
\end{tabular}

Tabla 1. Diferentes focos de los modelos de rehabilitación, integración y recuperación. Tomado de Saavedra (2009).

Desde el modelo de recuperación, las narrativas, autobiografías, informes, en definitiva, las experiencias subjetivas de personas con trastornos mentales graves (TMG), son esenciales. Son estas experiencias subjetivas las que nos dan cuentade procesos de recuperaciónexitosos y nos demuestran que, a pesar de la imagen 
ominosa de los TMG, es posible disfrutar de una vida llena de sentido y conectada socialmente. La defensa de la esperanza es un factor esencial en el modelo de la recuperación frente a las luctuosas expectativas del modelo biomédico que enfatiza el carácter crónico de los TMG. Esto no supone negar la enfermedad,sino privilegiar las historias de superación y de logro de una vida plena de muchos afectados. Historias que han sido apartadas e ignoradas por los relatos clínicos oficiales, lo que supone una segunda estigmatización (Ridgway, 2001). La recuperación implica acercarse a la salud mental desde el concepto de "sanar" y no desde el concepto de "curar" (Jacobson y Greenley, 2001). Es decir, aproximarse a la salud mental desde la recuperación va más allá de la remisión sintomática y no requiere necesariamente la vuelta al estado anterior a la enfermedad.

El núcleo del modelo de recuperación es la reconstrucción de la identidad de los afectados de forma que las experiencias de enfermedad se integren permitiendo que otros elementos de aquélla no sólo se desarrollen y no queden bloqueados, sino que adquieran nuevos significados y puedan ser compartidos socialmente. En este marco la percepción del propio control y el empoderamiento adquieren especial relevancia. El primero como recursos y estrategias de manejo del estrés y los síntomas. Y el segundo como toma del poder y, por lo tanto, responsabilidad. Capacidad para intervenir en el mundo, aceptar riesgos y hablar con voz propia (Jacobson y Greenley, 2001).

Este modelo se encuentra, como hemos visto, fuertemente basado en los movimientos de autoayuda que han exigido un cambio en los modelos de atención y en los métodos de investigación y, si cabe, una transformación epistemológica en salud mental. De tal forma que no sólo se consideren a las personas con TMG como sujetos experimentales en diseños de investigación, sino como generadores de conocimiento gracias a diseños cualitativos.

Aunque el contexto en el que nació el modelo de recuperación es muy específico y presenta un fuerte contenido reivindicativo e incluso político, se encuentra conectado con otros constructos que están configurando las prácticas y la organización de servicios en ámbitos distintos al de la salud mental. Por ejemplo, el modelo de "calidad de vida" en personas con discapacidad intelectual presenta dimensiones comunes con el de recuperación. Por citar algunas de estas similitudes, tanto el modelo de recuperación como el de calidad de vida de Schalock y Verdugo (2007) son aplicables a personas con o sin discapacidad o trastornos psicológicos, conformando un paradigma comprehensivo útil para todos los seres humanos. En el modelo de calidad de vida de Shalock y Verdugo (2007) los componentes objetivos son muy importantes, pero, al igual que en el modelo de recuperación, las experiencias subjetivas de los afectados son fundamentales. Tanto un modelo como otro descentran la atención de los problemas patológicos para enfatizar la necesidad de lograr la autonomía y el control de la propia vida.

El modelo de recuperación es recientemente conocida fuera del ámbito anglosajón, no obstante se puede encontrar un análisis histórico y un repaso de la investigación al respecto en revistas españolas (Saavedra, 2011). Este acercamiento a la salud mental, como veremos en el siguiente apartado, ha permitido una nueva configuración e implementación de las prácticas creativas en el ámbito de la salud mental. 


\section{La práctica creativa como "Recuperación Mutua"}

En nuestra opinión el concepto de práctica creativa presenta algunas ventajas al de arte-terapia en el marco de su utilización con personas con diversidad funcional o trastornos psicológicos, especialmente en recursos socio-sanitarios públicos. Podríamos decir que los dos conceptos son compatibles, pero que el primero reconfigura al segundo en algunos aspectos.

La práctica creativa se puede entender como cualquier acción que se ejerce sobre un objeto, desde el propio cuerpo hasta cualquier material, con el objetivo de transformarlo y dotarlo de nuevos significados. Es siempre de carácter voluntario y tiene un carácter lúdico y de diversión en cuanto abre un espacio simbólico diferenciable del resto de actividades. Este carácter de juego lo diferencia de cualquier actividad profesional, ocupacional o dirigida a algún fin instrumental.La práctica creativa se fundamenta siempre en la tradición socio-cultural de una comunidad, aunque se quiera transformarla y retarla. Del mismo modo que existen reglas en cualquier juego, las cualessiemprese pueden transformar. Entendido así, cualquier persona que realice una práctica creativa se encuentra en contacto con los valores y con la tradición cultural de una comunidad. En el ámbito de la salud mental esta idea nos parece esencial, ya que nos conecta a todos con una comunidad y acaba con la idea de creador salvaje, primitivo o genial, que provoca que se agrave el aislamiento de las personas que padecen alguna enfermedad o discapacidad. Dicho así, la práctica creativa tiene un marcado carácter comunitario, al menos como la entendemos nosotros, ya que o se lleva a cabo en conjunto, con un grupo o comunidad, o se ofrece para ser disfrutada como público, que no tiene que ser un público pasivo. El concepto de arte-terapia se encuentra muy conectado a las artes plásticas, pintura y escultura. Sin embargo, la práctica creativa se abre a cualquier actividad con las características antes comentadas: desde el teatro, pasando por la literatura y llegando a la música.

A nuestro entender, la práctica creativa en el ámbito de la salud mental incide en tres aspectos esenciales. La práctica creativa puede romper barreras sociales y conectar comunidades. En este sentido, las actividades artísticas de las personas con TMG o diversidad funcional, deben salir fuera de los recursos específicos o de la práctica individual para fundirse en la comunidad. La práctica creativa debe ayudar a todas las personasa reconstruir su identidad tal como lo describíamos anteriormente (Crawford, Brown, Baker, Tischler y Abrams, 2015; Saavedra, Cubero, Crawford, 2009; Saavedra y Rodríguez, 2008; Saavedra, 2009). Y desde luego, ayudar a expresar $\mathrm{y}$ entender experiencias y emociones.

Reformulando las palabras de Clemenceau referidas a la guerra podríamos afirmar que la salud es demasiado importante para dejarlas en manos de los médicos, que la salud mental es demasiado importante para dejarla en manos de psiquiatras, psicólogos y terapeutas y que, incluso, el arte es demasiado importante para dejarlo en manos de artistas.

El concepto de práctica creativa puede ser encuadrado en la tradición de las "Health Humanities". Utilizamos el término en inglés ya que no existe un término en español que abarque su significación. El profesor Paul Crawford (Crawford, Brown, Baker, Tischler y Abrams, 2015), principal valedor de este modelo, sintetiza en los siguientes puntos los retos de las "Health Humanities". Una nueva aproximación 
pedagógica a la formación de los profesionales implicados en los cuidados sociosanitarios que incluye el profundo conocimiento sobre la experiencia humana que nos facilitan las artes y las humanidades. Énfasis especial en los beneficios en términos de salud psicológica de la participación en actividades creativas por parte de los cuidadores familiares, cuidadores profesionales y usuarios de los servicios sociosanitarios indistintamente.Un esfuerzo por demostrar la eficiencia y la eficacia de estas prácticas en la mejora de la salud psicológica de las naciones. Un incremento de los recursos y capacidades de los cuidadores profesionales, familiares y afectados en la creación de nuevos contextos de cuidado. Y por último, un esfuerzo pordemocratizar las intervenciones "terapéuticas" cuando sea posible más allá de las profesiones especializadas.

Las "Health Humanities" pretenden recuperar "el significado" como objeto de estudio e intervención en las ciencias de salud. El significado ha sido marginado de las ciencias de la salud y abandonado a otras disciplinas como por ejemplo la antropología, la filosofía y la psicología cultural (Bruner, 1990;Giddens, 1991). La construcción del significado puede ser entendida como los procesos por el cual los humanos incardinan su experiencia subjetiva en marcos culturales y sociales dentro de una comunidad de forma que estas experiencias puedan ser comunicadas, narradas y compartidas, adquiriendo así sentido y finalidad. Este proceso es siempre dinámico, situacional y dialógico (Stetler, 2010). Una de las premisas esenciales de las "Health Humanities" es que las artes y las humanidades nos pueden ayudar a comprender y elaborar de forma óptima los significados de nuestras experiencias de salud, enfermedad y cuidado. Además, de acuerdo con la evidencia científica, la construcción de significados, lo que algunos autores han llamado coherencia,media nuestra respuesta fisiológica, siendo esencial en nuestra respuesta al estrés y por lo tanto determinante esencial de nuestra salud (Antonovsky, 1979).

Una pregunta esencial desde la perspectiva de las "Health humanites" es la siguiente: ¿Quién recupera a quién dentro del proceso creativo? Desde una perspectiva tradicional el paciente siempre es el recipiente de los cuidados, de la atención y de las técnicas de los especialistas. Cualquier intervención, incluso una actividad creativa, podría considerarse desde esta perspectiva. Sin embargo, una práctica creativa diseñada desde la recuperación mutua convierte el impacto en bidireccional. Los efectos de la actividad creativa se producen también en familiares, cuidadores profesionales, voluntarios y ciudadanos en general que pueden participar en la actividad. De este modo, los llamados pacientes se convierten también en cuidadores. Sin lugar a dudas, la práctica creativa basada en las "Health Humanities" se beneficia del impulso de lo que ha sido denominado "la nueva ola de la mutualidad" (Murray, 2012).

Como afirmamos al final del primer apartado, es necesario evaluar el impacto de las prácticas creativas en la salud psicosocial. Secker, Hacking, Spandler, Kent y Shenton(2007) diseñaron una ambiciosa investigación integrando metodología cualitativa y cuantitativa de 102 prácticas creativas con personas con trastorno mental grave. En un estudio de casos de carácter cualitativo que se incluía la investigación, todos los participantes de los talleres refirieron mejoras en tres procesos psicológicos: Motivación, concentración y conexión con otros. Algunos de los participantes señalaron además como efectos positivos derivados de las prácticas creativas la expresión de emociones y experiencias, la adquisición de habilidades y el hecho de 
estar fuera de entornos estresantes.Así, los autores hallaron evidencia empírica de mejora del bienestar psicológico, el empoderamiento y de la inserción social de los participantes.

\section{Dos ejemplos de Práctica creativa como recuperación mutua}

Dentro del proyecto de investigación "Creative Practice as Mutual Recovery: Connecting Communities for Mental Health and Well-Being" liderado por el "Centre of Social Futures" del "Mental Health Institute" de Nottingham y financiado por el "Royal Arts and Humanities Council", nos hemos propuesto evaluar por primera vez el impacto de dos prácticas creativas con grupos vulnerables en la ciudad de Sevilla. En este apartado describimos brevemente estas prácticas creativas y el procedimiento de evaluación que actualmente se está llevando a cabo.

El objetivo de la evaluación presenta tres dimensiones de análisis.La primera el grado en que los usuarios (personas con TMG o personas diagnosticadas con Síndrome de Down (SD) se benefician de la actividad en términos de bienestar psicológico, inclusión social y recuperación, entendida ésta última tal como fue definida más arriba.

La segunda el grado en que la participación en estas actividades beneficia a los monitores, profesionales o voluntarios que comparten las actividades con los usuarios también en términos de salud psicológica. En este caso, es muy interesante explorar cómo se transforman las imágenes de la enfermedad o de la diversidad funcional de estos profesionales.

Por último, queremos evaluar el impacto de la actividad en las instituciones de las cuales residen los usuarios y que soportan la actividad. ¿Cambian las prácticas profesionales? ¿Existen cambios en las políticas? ¿Afecta en algún grado a la organización de esas instituciones?

\subsection{Talleres creativos en el Museo de Arte Contemporáneo de Sevilla}

El Centro Andaluz de Arte Contemporáneo (CAAC) fue fundado en el año 1990 con la intención de dotar a esta Comunidad Autónoma de una institución apropiada para la investigación, conservación, promoción y difusión del arte contemporáneo. En el año 1996 se trasladó al antiguo Monasterio de la Cartuja de Sevilla y se convirtió en un organismo autónomo dependiente de la Consejería de Cultura. Desde el año 2006 y gracias a un convenio entre el Servicio Andaluz de Salud (SAS), la Dirección del CAAC y la Fundación Pública para la Integración Social de las personas con Enfermedad Mental (FAISEM)un grupo de personas con Trastorno Mental Grave (TMG) disfrutan seminarios creativos en las instalaciones del CAAC.

En concreto se celebran cada año tres seminarios de seis sesiones cada uno. Normalmente cuatro grupos de entre 10 y 15 usuarios de distintos dispositivos participan por separado una vez a la semana en cada seminario. Los dispositivos de los que provienen los usuarios son comunidades terapéuticas, unidades de rehabilitación, casas hogares, talleres ocupacionales, la asociación de familiares, allegados y personas con trastorno mental grave. También participaron durante algunos años internos del hospital penitenciario y este año, 2015, se realizará una 
experiencia piloto con jóvenes de la unidad de salud mental infantil de dos hospitales sevillanos.

Una característica esencial de esta actividad,desde nuestra perspectiva, es que se aprovecha en cada momento la exposición temporal que exhibe el museo, sea cual sea el motivo o las técnicas de la misma, para dotar de contenido a los talleres. Por otra parte, la actividad se desarrolla en el museo y en muchos momentos los espacios son compartidos por el público que visita el mismo. De este modo se integra la actividad en un contexto espacio-temporal normalizado y se "obliga" a los usuarios a salir de sus dispositivos. Las sesiones son dirigidas por una mediadora artística con experiencia con la población que propone actividades y sugiere su contenido, siempre utilizando las exposiciones temporales. Varios monitores, empleados de los recursos de donde vienen los usuarios, acompañan a los usuarios y participan en las actividades en todas las sesiones de los seminarios.

Las seis sesiones en las que se componen cada seminario-taller se dividen en dos partes. La primera una visita participativa a la exposición temporal en que se propone un trabajo de mediación artística. La mediación intenta facilitar la comunicación entre cada usuario y la obra promoviendo una interpretación libre de acuerdo con la experiencia de cada uno de ellos. Posteriormente se planifica un trabajo de producción artística en el que, utilizando contenidos de la exposición, se trabaja individualmente o colectivamente utilizando variadas técnicas.

En la siguiente página existe amplia información sobre los orígenes, metodología y un resumen con imágenes de las obras de los usuarios de la mayoría de los seminarios.

http://www.juntadeandalucia.es/servicioandaluzdesalud/principal/ documentosacc.asp?pagina $=$ gr_smental_derrecup_4

Entre las exposiciones sobre las que se ha trabajado y de las cuales se puede obtener información en la anterior página web podemos encontrar: Prohibido el cante. Flamenco y fotografía, del 5 de mayo al 12 de junio de 2009; Máquinas de mirar o cómo se originan las imágenes, del 2 de noviembre al 15 de diciembre de 2009; Los Esquizos. Pintura figurativa madrileña de los 70, del 9 de febrero al 19 de marzo de 2010; AiWeiwei. Resistencia y tradición y Nacho Criado. Agentes colaboradores, del 12 de febrero al 22 de marzo de 2013.

En la actualidad estamos evaluando uno de los seminarios (en total 60 usuarios con TMG y ocho monitores). Se consideraron tres estrategias para obtener datos de forma que podamos triangular los nuestro resultados como método de fiabilidad. Primero,tanto usuarios como monitores cumplimentarán dos cuestionarios, pre-test y post-test, para evaluar su bienestar psicológico y su inclusión social. Estamos utilizando el Warwick Well being Questionnaire (versión española, López, et al. 2013) y el Secker Social Inclusion Questionnaire (Secker, Hacking, Kent, Shenton y Spandler, 2009). Éste último lo hemos traducido para la ocasión. Segundo, entrevistaremos a una muestra de usuarios y monitores mediante una entrevista semi-estructurada. También entrevistaremos a la mediadora artística y a los fundadores de la iniciativa por parte de los Servicios de Salud. Por último, hemos incluido en cada grupo un estudiante de psicología pero sin experiencia en salud mental para que participe activamente al tiempo que también observe las dinámicas en los talleresbasándonos en la técnica de investigación-acción. Las experiencias y las impresiones de cada uno se analizaran mediante un grupo de discusión de acuerdo con las sugerencias del reciente informe 
"The value of the Arts in Therapeutic and Clinical Interventions: A critical review of the literature" (Bungay, Munn-Giddings, Boyce, and Wilson, 2014), en el cual se apuesta por técnicas de investigación participativas.

\subsection{Actividad del grupo de teatro de ASEDOWN}

La asociación de Síndrome de Down Asedown-Sevilla es una asociación que tiene como misión favorecer, desarrollar y propiciar todo tipo de actividades, programas y servicios, encaminados a potenciar la plena integración familiar, escolar y social de las personas con Síndrome de Down (SD) y sus familiasconsiguiendo mejorar la calidad de vida de estas personas.http://www.asedown.org/index.html

Inicialmente el proyecto del grupo de teatro nace de la idea de estudiantes de psicología que se pusieron en contacto con la asociación para desarrollar una clase de teatro. Este proyecto no tuvo éxito y la profesora de la clase del grupo de autogestores (otro grupo de la asociación) adoptó la idea y decidió que en sus clases dedicaría parte de su horario a impartir clases de teatro. Para ese entonces (1996), no había un grupo determinado de teatro, sino que en esta clase, participaban todos lo que asistían a clases en la asociación. Se realizó una representación como resultado de esas clases y resultó tan satisfactorio que el proyecto se consolidó.

Es entonces cuando la asociación ASEDOWN establece un convenio con el Colegio Maristas San Fernando para poder utilizar su espacio destinado al salón de actos y desarrollar el curso de teatro, que tiene una duración de 9 meses y una frecuencia de dos horas a la semana. Debido al poco tiempo que se tiene destinado para las clases de teatro y lo mucho que hay que repetirpara poder lograr unresultado final, las actividades del grupo de teatro van muy ceñidas a lo que son las prácticas de ensayo. En algunas ocasiones se pueden hacer juegos teatrales como los de identificación de sentimientos, el espejo y calentamiento del cuerpo.

Esta actividad de teatro se desarrolla gracias a la colaboración de un grupo de voluntarios, entre ellos están tres actores profesionales que se unen a la representación como colaboración. Cuatro estudiantes de bachillerato del colegio Maristas, que actúan y a la vez trabajan como monitores para el mejor desarrollo de los ensayos. Por ejemplo,reforzando algunos personajes que lo necesiten. El grupocuenta con diez integrantes con diferentes grados de diversidad funcional. Dos jóvenes de parálisis cerebral (PC) y ocho adultos con SD, con edades entre 23 y 40 años. La edad media es de 27 años. El curso finalizacon una representación final abierta al público en la cual se suele incluir un número musical.

Para evaluar este proyecto hemos planteado un diseño esencialmente cualitativo. Hemos diseñado cuatro entrevistas semiestructuradas para cuatro grupos de personas explorando los puntos indicados al principio de este apartado. Una entrevista para los padres de las personas con SD, otra para éstos, nos gustaría entrevistar a los voluntarios del colegio y por último, una entrevista especialmente diseñada para la directora y fundadora del grupo. Además, una alumna de doctorado especialista en teatro, pero sin experiencia previa con personas con $\mathrm{SD}$, se integrará como observadora-participante en el grupo de teatro.

Todas las entrevistas fueron diseñadas grupalmente en seminarios formados por profesores y alumnas de doctorado y grado especialmente interesados en la 
práctica creativa. En los citados seminarios se estudiaron los objetivos del proyecto de investigación original, se analizó la literatura existente, y se ofreció una primera aproximación a las experiencias a estudiar por las personas que establecieron el primer contacto con las instituciones.

Debido a que todos sus integrantes llevan años participando en el taller de teatro no tiene sentido un estudio pre-post. No obstante, gracias a la existencia de escalas validadas y estandarizadas especialmente diseñadas para personas con discapacidad intelectual o del desarrollo que estudian la calidad de vida, planificamos explorar la calidad de vida de los participantes afectados con SD y PC al final del taller de teatro y compararlo con la población normativa. En concreto, seleccionamos la escala INICO-FEAPS (Evaluación Integral de la Calidad de Vida de personas con Discapacidad Intelectual o del Desarrollo) de Verdugo et al. (2013), que se compone de ocho factores y presenta dos sub-escalas: un auto-informe y un informe de terceras personas.

\section{Reflexión final}

Las prácticas creativas descritas brevemente presentan tres características que las hacen compatibles con el modelo que hemos intentado defender en el presente artículo y, por lo tanto,idóneas para nuestra investigación.Primero, se llevan a cabo en ámbitos totalmente normalizados fuera de cualquier contexto sanitario o social especializado. De hecho, los espacios donde se llevan a cabo son compartidos por otras actividades y personas de la comunidad en general. Segundo, en ellas participan, al menos en un principio, tanto personas afectadas como voluntarios y profesionales. Es decir, no son actividades focalizadas en exclusiva para un grupo de afectados por alguna enfermedad o característica especial. Y tercero, los objetivos de estas prácticas creativas no son explícitamente la disminución de la sintomatología, obtener un diagnóstico, la mejora cognitiva o cualquier otro fin clínico, sino de forma abierta incrementar la participación de grupos vulnerables en actividades culturales que aumenten su inclusión social y su bienestar psicológico.

Es importante señalar que las prácticas creativas desde esta perspectiva no están diseñadas exclusivamente para personas afectadas, sino que es igual de importante el impacto de la práctica en voluntarios, profesionales o participantes de la comunidad. En nuestra opinión, la práctica creativa como recuperación mutua es una buena herramienta de mejora de la calidad de vida ya que sitúa a la persona con discapacidad o con un TMG más allá del rol de recibidor de cuidados o usuario de servicios. Es decir, estas personas también se convierten en cuidadores, agentes activos de procesos de recuperación y mejora de la calidad de vida de todas personas participantes en la práctica creativa. Estas prácticas presentan un triple impacto directo o indirecto en la calidad de vida de las personas. En un nivel individual, ayudando a mejorar algunas dimensiones de la calidad de vida como el bienestar psicológico, la inclusión social, desarrollo personal, etc., de todos los participantes. Por otra parte, una transformación de la imagen de uno mismo a través de la mirada de los otros. Es especialmente importante estudiar cómo se transforma la imagen que tienen profesionales y voluntarios de las personas afectadas tras participar conjuntamente en prácticas creativas, y qué repercusión tiene este cambio de imagen en la identidad 
de los afectados. Y por último, un reto institucional que puede conllevar cambios organizativos y de prácticas profesionales en las instituciones y asociaciones en cuyo seno se realizan las prácticas creativas.

Esperamos confirmar en los análisis actualmente en curso que la práctica creativa tal y como la entendemos funciona como una herramienta de inclusión social y reconstrucción de significados eficiente en personas especialmente necesitadas de ello. Y además, puede aumentar el bienestar psicológico, más allá de una simple reducción de la sintomatología propia de enfermedades y síndromes, en población española, tal y como se ha hecho en otros países. En la misma línea, también esperamos explorar los procesos psicológicos que hacen que esto sea posible.

\section{Agradecimientos}

Quisiéramos explícitamente dar las gracias a la dirección del Museo de Arte Contemporáneo de Sevilla, al Servicio Andaluz de Salud y a la Fundación Andaluza para la Integración Social de las Personas diagnosticadas con Enfermedad Mental (FAISEM). Además, agradecemos la colaboración a todos los usuarios y monitores de los dispositivos que participan en la actividad. En especial a Felipe Vallejo, uno de los iniciadores del proyecto, y a Sandra Melgar Sevillano mediadora artística que coordina los seminarios. También agradecemos a ASEDONW y a su grupo de teatro liderado por Pilar por abrirnos desde el principio las puertas y permitirnos integrarnos en su grupo. Muchas Gracias a todos y todas.

\section{Referencias}

Antonovsky, A. (1979). Health, Stress and coping. San Francisco: Jossey-Bass. Bruner, J. (1990). Acts of meaning. Cambridge, MA: Harvard University Press.

Bungay, H., Munn-Giddings, C., Boyce, M., and Wilson, C. (2014). The value of the Arts in Therapeutic and Clinical Interventions: A critical review of the literature. AHRC Cultural and Values Project.

Crawford, P., Brown, B., Baker, C., Tischler, V. y Abrams, B. (2015). Health Humanities. Palgrave McMilliam: New York.

Fichte, J.G. (1984).Segunda introducción a la Teoría de la Ciencia. Madrid: Sarpe.

Giddens, A. (1991). Modernity and self-identity: Self and society in the late modern age.Stanford CA. Stanford UniversityPress.

Gilman, S.L. (2001). Los locos como artistas. EnM. Dávila (Coord.) La colección Prinzhorn. Trazos sobre el bloc mágico. Barcelona: MACBA.

Hill, A. (1943). Art as an aid to illness: an experiment in occupational therapy. London: Studio magazine.

Jacobson. N. y Greenley, D. (2001). A conceptual model of recovery. Psychiatric Services, 52(5), 688-688. doi:10.1080/09638230701677803.

Jaspers, K. (1913). Allgemeine Psychopathologie. Ein Leitfaden für Studierende, Ärzte und Psychologen.Berlin: Springer.

Laing R.D. (1960). The divided self (a study of sanity and madness).London: Tavistock Publications. 
López, M.A., Gabilondo, A., Codony, M., Garcia-Forero, C., Vilagut, G. y Castellvi, P. (2013). Adaptation into Spanish of the Warwick-Edinburgh Mental Well-being Scale (WEMWBS) and preliminary validation in a student sample. Quality of Life Research, 22, 1099-1104.doi: 10.1007/s11136-012-0238-z.

Murray, R. (2012). The new Wave of Mutuality: Social Innovation and Public Service Reform. London Policy Network paper.

Prinzhorn, H. (1922). Bildnerei der Geisteskranken. Berlín: Springer.

Reynolds, M.W., Nabors, L. y Anne Quinlan, A. (2000). The Effectiveness of ArtTherapy: Does it Work? Art Therapy: Journal of the American Art Therapy Association, 17(3), 207-213.doi:10.1080/07421656.2000.10129706.

Ridgway, P. (2001). Restorying psychiatric disability: learning from first person recovery narratives. Psychiatric rehabilitation journal, 24(4), 335-343.

Saavedra, F.J. y Rodríguez, A. M. (2008). Dimensiones de la educación artística: Experiencias de un taller de pintura popular en un pueblo de Sevilla. Arte, individuo y sociedad, 20, 43-68.

Saavedra, F.J. (2011). Cómo encontrar un lugar en el mundo: explorando experiencias de recuperación de personas con trastornos mentales graves. História, Ciências, Saúde - Manguinhos, 18(1), 121-136doi:10.1590/S0104-59702011000100008.

Saavedra, F.J. (2009). Schizophrenia, narrative and change: Andalusiancarehomes as novel sociocultural context. Culture, Medicine and Psychiatry, 33(2), p.163-184. doi: doi:10.1007/s11013-009-9128-4.

Saavedra, F.J., Cubero, M. y Crawford, P. (2009). Incomprehensibility in the narratives of individuals with a diagnosis of schizophrenia. Qualitative Health Research, 19(11), 1548-1558doi: 10.1177/1049732309351110.

Secker, J., Hacking, S., Spandler, H.,Kent, L. y Shenton, J. (2007). Mental Health, Social Inclusion and Arts: Developing the evidence Base. Anglia Ruskin University and Department of Health. Recuperado el 25 de febrero de 2015 en:http://www. instituteofhealthequity.org/projects/mental-health-social-inclusion-and-artsdeveloping-the-evidence-base

SeckerJ., Hacking S., KentL., ShentonJ... y Spandler H. (2009). Development of a measure of social inclusion for arts and mental health project participants. Journal of Mental Health 18(1), 65-72.doi:10.1080/09638230701677803.

Stetler, R. (2010). Experience-based, body-anchored qualitative research interviewing. Qualitative Health Research, 20:6, 859-867.doi: 10.1177/1049732310364624.

Schalock, R.I. y Verdugo, M.A. (2007). El concepto de calidad de vida en los servicios y apoyos para personas con discapacidad intelectual. Revista española sobre discapacidad intelectual, 224, 21-36.

Verdugo, M.A., Gómez, L.E., Arias, B., Santamaría, M., Clavero, D. y Tamarit, J. (2013). Escala INICO-FEAPS. Evaluación Integral de la Calidad de Vida de personas con Discapacidad Intelectual o del Desarrollo. Salamanca. Publicaciones del INICO. Instituto Universitario de Integración en la Comunidad.

The Future Vision Coalition (2009). A future vision of mental health: The Future Vision Coalition. Recuperado el 17 de agosto de 2014 en:http://www.nhsconfed. org/ /media/Confederation/Files/Publications/Documents/Future_vision_for_ mental_health_FINAL.pdf 